\title{
Dynamic Generation of Drawings in Facility Management System
}

\author{
Makoto KIKUCHI \\ Senior Researcher, \\ Yuusaku YAGI \\ Facilities Management Systems, Transport Information Technology Div.
}

Mikio TANAKA, Dr. Eng.

General Manager,

Transport Information Technology Div.

\begin{abstract}
Many railway companies in Japan have adopted a computerized facility management system for better efficiency and other benefits. There are some serious problems, however, such as high costs of initial data registration and inconsistency between ledgers and drawings. In order to solve these problems, we have proposed a facility management system called Dynamic Generation System (DGS). Further, we developed a method to construct a database as a facility ledger, by arranging facilities on the drawing interactively and setting attribute data. We show an example of the method of data writing on drawings applied to electric power facilities.
\end{abstract}

Keywords : facility management system, drawings, Dynamic Generation System, database

\section{Introduction}

Railway facilities consist of various kinds of equipment, which have been managed by office workers with help of voluminous ledgers, drawings and diagrams. In the past decade, however, a number of railway companies in Japan have adopted a computerized facility management system for better efficiency and higher profits. Despite all these efforts, there are still serious problems such as high costs of initial data registration and inconsistency between ledgers and drawings. In order to solve these problems, we have developed a facility management system called $\mathrm{DGS}^{1)}$ that has the functions of ledger management and automatic drawing. The database of this system contains only ledgers and additional information which is described in a plain text format. Drawings are dynamically generated with those data. Therefore, there is no inconsistency between ledgers and drawings. This system has further merits. The software and the stored data are small and can easily be installed in portable computers. We can provide a user interface better than that of the existing system ${ }^{2}$. Therefore, drawings and ledger data can be referred to in field work. It provides more convenience for field engineers.

This system has been introduced into some railway companies to verify its remarkable merits and examine the feasibility of this system ${ }^{3}$. It is estimated that development costs of the system will be reduced to about half and a more effective use of stored data is expected.

\section{Problems on the existing facility management systems}

\subsection{High costs of initial data registration}

Most of the railway companies in Japan manage facilities by referring to not only ledger data but also drawing data which were initially hand-written on paper.
These enormous amounts of data have to be registered into a computer system in advance. Ledger data contains attributes of facilities, such as facility type, product type, manufacturer, implementation date and other data. Drawing data contain the existing positions of facilities, functional relations among facilities and shapes of facilities, etc. These data are mostly registered by computer aided design (CAD) operations such as image scanning, tracing and digitizing processes. The registration process requires enormous labor. In some companies in Japan, there were some 10 thousands of drawings which took almost a year for registration. Furthermore, this operation needs special equipment, such as a workstation with a mass storage device, an image scanner for scanning of drawings, a large-scale plotter to output drawings, and a dedicated software for translation from scalar data to vector data. Therefore, it is difficult to perform this registration process on plural computers at the same time, because some equipment is placed on only one unit from the viewpoint of cost performance and it requires skilled operators.

\subsection{Inconsistency between ledgers and drawings}

After the initial registration, the system will be used, referred to, and updated for daily work. Stored data will often be updated because of changes in the location, attributes and number of facilities, etc. Ledger data are often stored as text data under an ordinary database management system (DBMS) or on spreadsheets. Drawings are stored as CAD data separately. Both data are managed separately under different systems, and must be updated precisely and consistently by office workers, which are, however, sometimes neglected. It is a main reason why inconsistency between ledgers and drawings has often occurred. We know that, in most of these cases, the ledgers are usually renewed, but drawings are left as they are. As a result, many inconsistencies remain be- 


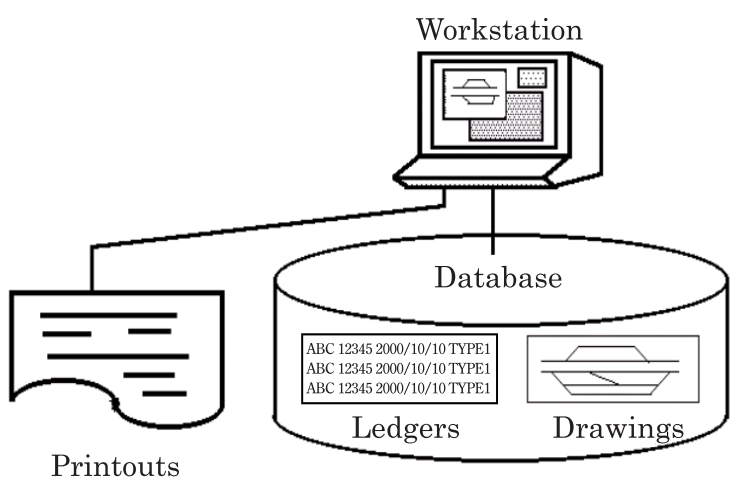

Fig. 1 Existing facility management system

tween ledgers and drawings. Afterward, they will cause problems, because they do not tell which data has correctly been updated. This may be natural because each system is operated by different operators and drawings are rarely updated, unlike ledgers. Figure 1 shows a data management method of the existing facility management system.

\subsection{Other problems}

A few railway companies use a hand-held computer with a flash ROM card to get some inspection data. Although the computer collects inspection data satisfactorily, it is not easy for field engineers to enter the data into the computer, because the computer does not have a sufficiently large size display to view drawings or sufficient power for CAD processing. Due to these problems, railway companies built menu-based systems. But it is not convenient in the case of deep hierarchy data structure. It takes a long time to get some necessary information.

In the field of tele-communications, there are no computerized systematic drawings which show relations among a number of multiplexers. Systematic drawings contain a huge number of relations which are changed frequently. Although it may be possible to build systematic drawings by using a CAD system, there is a problem that workers have to modify many CAD files which contain objects to update, even for a single modification. This modification work is also apt to cause inconsistency among CAD files. If we build a systematic drawing as only one CAD file, the view area which includes logical coordinates will become excessively large. We think that the size of display equipment is too small to display the view area and the scroll function does not fit.

\section{Features of DGS}

\subsection{Aims of DGS}

As mentioned above, the existing systems have some serious problems. We consider that they are mostly due to the data saving method. Inconsistency between ledger data and drawing data can not be solved unless these data are unified. In order to solve the above-mentioned problems, we have developed a system called DGS. In this system, the database contains only ledgers and additional information which is described in a plain text format.

\begin{tabular}{|c|c|c|c|c|}
\hline Drawing 1 & near X station & & 123.456 & 123.789 \\
\hline Track1 & Down Main & & & \\
\hline Track2 & Down 1st & Down Main & 123.524 & 123.698 \\
\hline Track3 & Down 2nd & Down 1st & 123.602 & 123.755 \\
\hline Track4 & Up Main & & & \\
\hline & ... & $\cdots$ & $\cdots \cdots$ & \\
\hline Facility1 & Signal1 & Down Main & 123.655 & \\
\hline$\cdots$ & $\cdots$ & $\cdots$ & ...... & $\cdots$ \\
\hline$\cdots \cdots$ & $\ldots \ldots$ & $\ldots \ldots$ & $\cdots \cdots$ & $\cdots \cdots$ \\
\hline Facility 4 & Point & Down Main & 123.688 & \\
\hline
\end{tabular}

Fig. 2 An example of track descriptions

Drawings are dynamically generated with those data. So, there is no inconsistency between ledgers and drawings.

In addition, we consider how drawings can be generated. There are a number of cases, where workers refer to drawings with particular interest. They do not refer to the whole drawing, but a part they need. Therefore, it is not necessary to generate whole drawings. It is enough to generate a part of drawing. After all, it is possible to display a huge drawing on display equipment. This system has a further merit. The software and the stored data are small enough to be installed on portable computers easily. Therefore, drawings and ledger data can be referred to in field work. An example is shown in Figs. 2 and 3 . We expect that most of the problems contained in the existing drawing system can be solved by this system.

\subsection{Reduction of initial data registration}

At railway companies, there are a great number of drawings and diagrams which have been initially handwritten, and have to be registered into the facility management system in advance. There have been two ways to register these drawings. One is to use a data scanner, and the other to utilize the CAD tool. In any case, these two ways require special equipment which is expensive and manipulated by special operators. In addition, it takes a long time to complete registration. In the case of facility management system with DGS, only the ledger data with some additional information, which indicates positioning location for generating drawings, are to be registered. These data are mostly extracted from the existing ledger, and it takes far less time to complete

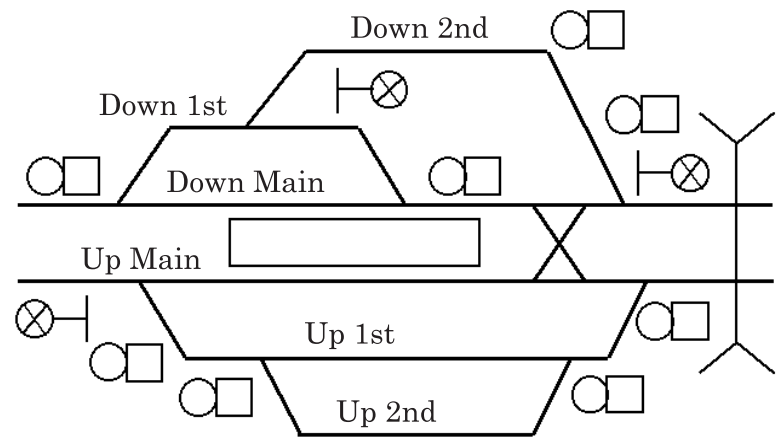

Fig. 3 An example of drawing by DGS generation 


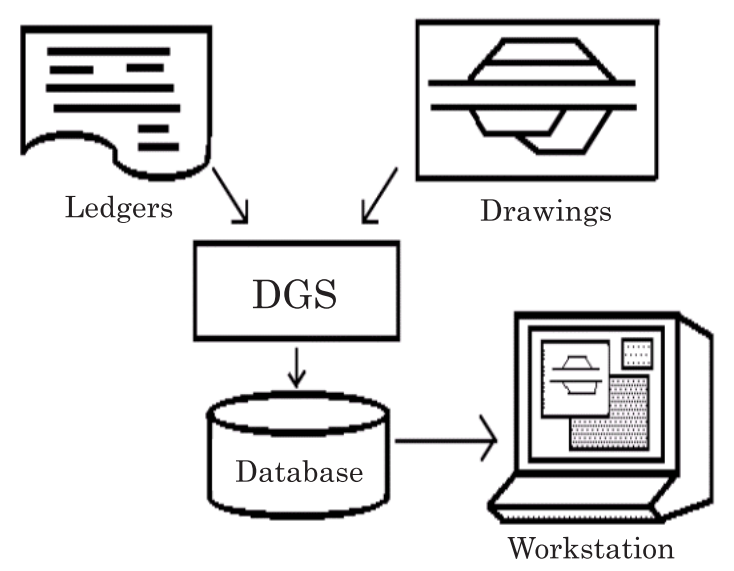

Fig. 4 Facility management system with DGS

extraction. It is comparatively effortless to register it into the system. As the format of this data is the text type, therefore, the data can be registered without special equipment or skilled operators.

\subsection{Elimination of data inconsistency}

DGS offers functions to generate drawings dynamically only by referring to ledger data. DGS has no statistic drawing data. The algorithm of DGS has been elaborated to enable users to view drawings. DGS can generate and display them almost immediately.

There often occur changes concerning facilities, such as the type, manufacturer, location, and other data. Although these changes must be followed precisely and immediately by office workers, it is often delayed or neglected. In the case of DGS, as long as the ledger is updated correctly, the related drawings viewed after the update have automatically been conducted. Users can get the latest status of drawing all the time. There would be no inconsistency in the case of facility management system with DGS. Unified data which are managed by DGS are shown in Fig. 4. In addition, workers can refer to the newest drawings any time. In many cases, data update of drawings takes place just a few times a year. Compared with the management method of traditional system, therefore, that of DGS seems to be far better.

\section{Flow of dynamic generation process}

DGS deals with text format data. Contents of database are not fixed, but have various description types. An approximate flow of dynamic generation is shown below.

\subsection{Appointment of viewing}

When users want to refer to some drawings, they have to appoint a display area at first. After that, DGS starts a process to read data from the database to a memory. The above process is applied only to generating information. Contents of appointment have various types, such as the kilometer post in signaling system drawings and a particular multiplexer or a particular path name in transmission system drawings.

\subsection{Drawing of base facility}

After the reading process, DGS generates a base facility by using a part of attribute data. It means that it is sometimes positioning information in a display, or a kind of facility at other times. There are various methods to indicate facility symbols. There are some cases where a particular font is used or particular symbols are generated from a part of database.

\subsection{Drawing of other facility}

Most of other facilities are frequently managed by the relation with the base facility. After drawing the base facility, therefore, DGS automatically generates other facility layers by using the above relational information. There are some cases where it can generate additional symbols by user's appointment.

\subsection{Implementation samples of drawing generation}

Figure 5 shows a signaling facility drawing by DGS. Recently, it is normally the case to display this kind of drawing on a computer. But in this case, there is a remarkable difference that a program generates a drawing directly by using attribute data of facilities registered by each element in the drawing, without $\mathrm{CAD}$ or image data scanning. If attribute data of a facility is changed, then, the generated drawing will be changed automatically.

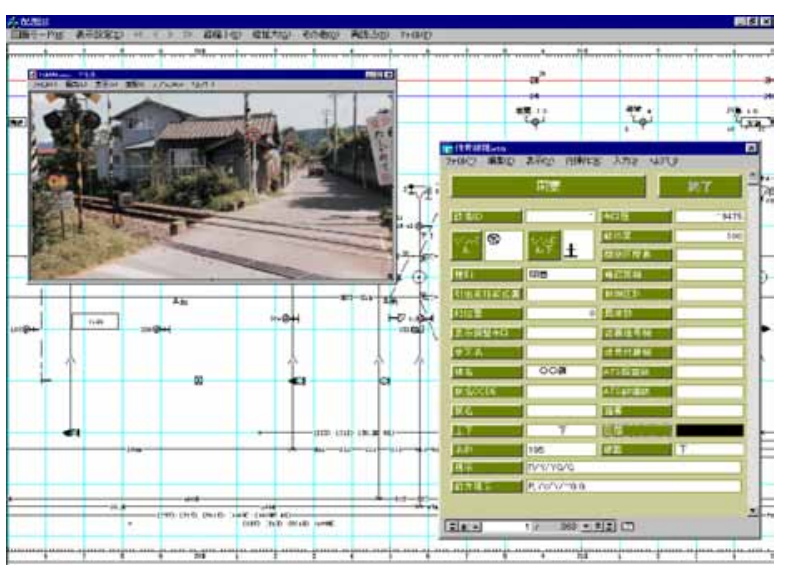

Fig. 5 Signaling facilities drawing by DGS

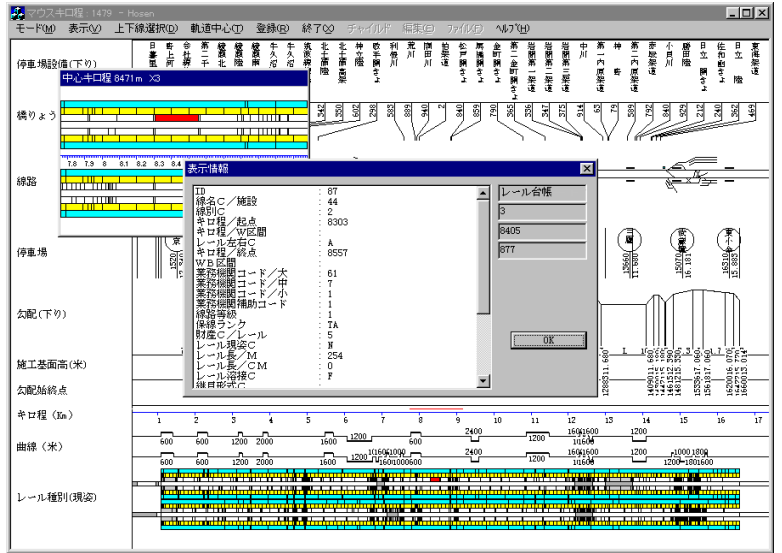

Fig. 6 Track maintenance facilities drawing by DGS 
Figure 6 shows a management drawing of track maintenance facilities including a layout of alignment, grade and curve of rails, and information about rail, crosstie and ballast. There is an area which shows classification of rail, crosstie and ballast at the bottom of the drawing, and it can be displayed with an enlargement in another window. Usually, this kind of drawing was generated approximately once a year by a hand-writing method or $\mathrm{CAD}$. In this case, however, a program generates a drawing by using ledger data.

\section{Correspondence to data construction function}

\subsection{Registration and change of facility}

DGS by the facility description language which we have dealt with up to this point needed to be provided with facility ledgers as a database.

In the case that there are no ledgers for facilities management by the reason that it was a newly open line, however, it is impossible to generate drawings as usual. Therefore, we have developed additionally a function to input facility data directly on the drawing. More specifically, we established a display mode in which it generates facility drawings as usual by using data already registered in the ledgers and an edit mode in which it newly registers or changes a facility. In the case of registration of facility, DGS also shows a sub-window for facility registration on the drawing, takes in data interactively, and further writes data into the database.

Thus, it has become possible to perform the following transaction interactively and visually.

(1) Registration of new facility.

(2) Changing the classification of existing facility.

(3) Changing the location or attribution of existing facility.

As an example of applying these new functions, we also developed a dynamic generation system for drawings concerned with electric power facilities. On that occasion, for simplification, we divided facilities into spot facilities and linear facilities, and applied different registration ways for these facilities. Figure 7 shows a pic-

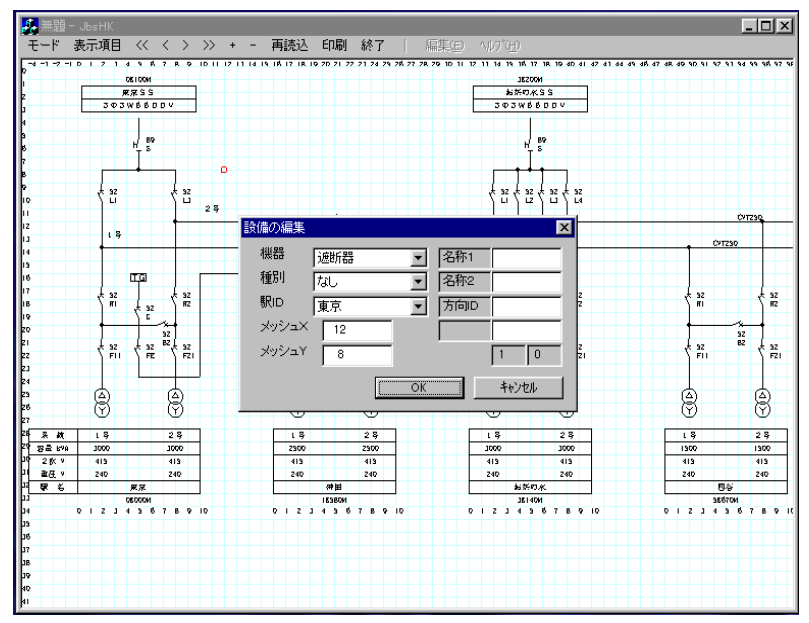

Fig. 7 Registration of a facility ture of registration of a facility.

\subsection{Actual drawing generation}

(1) Systematic drawing of power distribution

The systematic drawing of power distribution is a drawing which shows a route from substations to transformers in each station, and indicates the following facilities.

- Substation, circuit breaker, disconnect switch, transformer, rated output of each station, and their power distribution route.

The systematic drawings of power distribution is generated by arranging facilities not according to kilometers of rails but according to positional relation between stations and substations. So, we determined to draw crosswise meshes on the drawing and define the position of a facility with $\mathrm{x}$-coordinate and $\mathrm{y}$-coordinate.

(2) Trolley wire plan

The trolley wire plan is a drawing which shows a trolley wire and surrounding equipment, grade and curve data of rails, and a traction supply system, and indicates the following facilities.

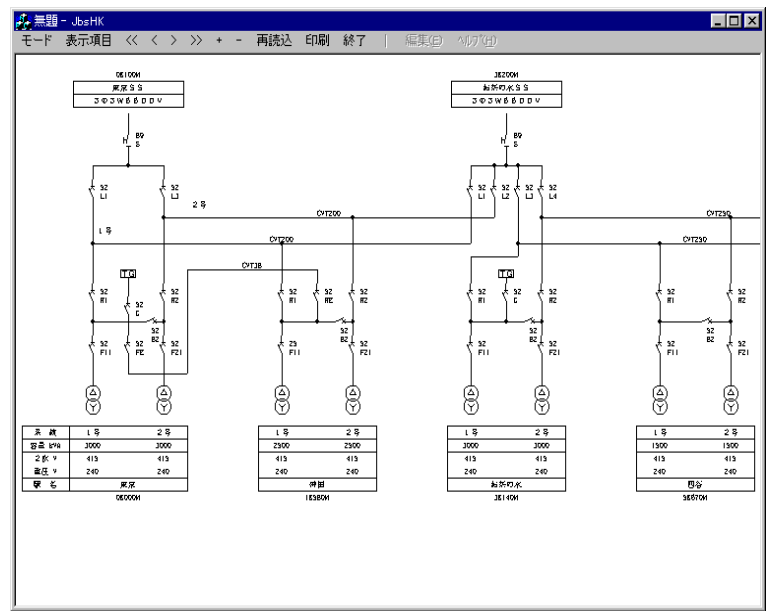

Fig. 8 Systematic drawing of power distribution

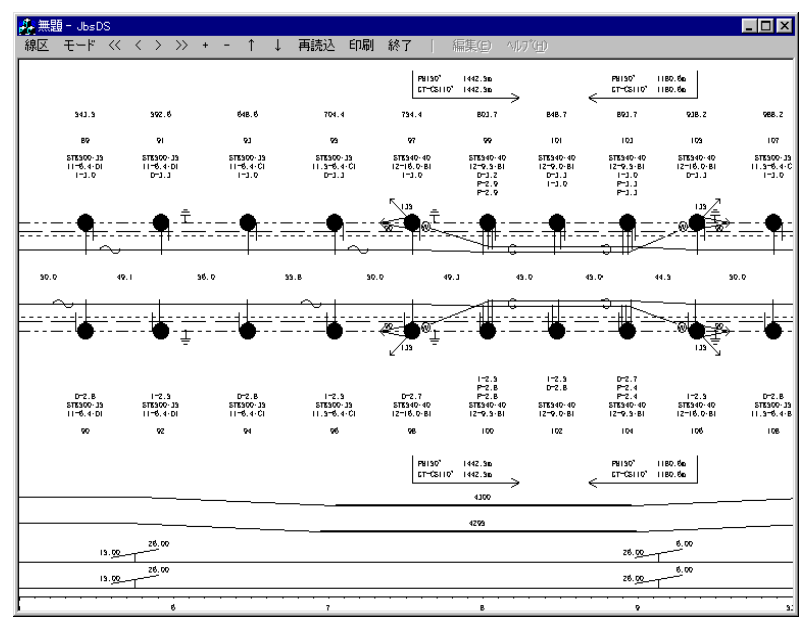

Fig. 9 Trolley wire plan 


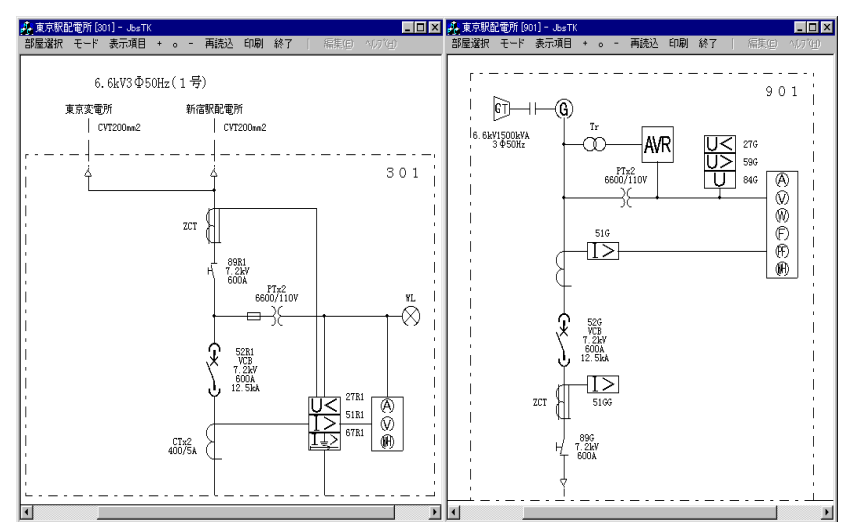

Fig. 10 Single line circuit diagram

- Pole, trolley wire, feeder line, bracket, section break, steady brace, balance weight, connector, etc.

In this drawing, poles and trolley wires are arranged according to kilometers of line, and each facility such as a bracket and a balance weight is defined as a subordinate to a pole.

(3) Single line circuit diagram

The single line circuit diagram is a drawing which shows the electric connection of facilities in a substation or a switching station, and indicates the following facilities.

- Circuit breaker, current transformer, rectifier, relay, and their wiring.

In the same way as the systematic drawing of power distribution, we define in this drawing the arrangement of each facility in a room by $\mathrm{x}$-coordinate and y-coordinate on mesh.

Moreover, it is possible to register and change a facility interactively on any drawing.

\section{Benefits of DGS}

Although there are some exceptions, many kinds of drawings suit this system and bring more benefits as mentioned above than the existing systems. We have not yet verified quantitative evaluation sufficiently, but succeeded in verifying remarkable merits in qualitative evaluation as follows.

\subsection{High cost/performance}

DGS can run on a personal computer without requiring expensive equipment. Therefore, stored data are available for use on most equipment even with a handy terminal. Some railway companies have already built a database for facility management. So, we registered the additional data for DGS. As a result, they can easily use the data which are stored in their workstation on every personal computer, and it brings about more benefits for field engineers.

\subsection{Speedy registration of initial data}

Data are initially registered without requiring specialists in the scanning and tracing processes. This work can be performed at the same time by a number of personal computers. This is the most important advantage of the newly developed system. We have already introduced this method and have implemented initial data registration. In many cases, ledger data has already been built in systems. As a result, we have to build drawings data as additional information because of isolation from existing ledger data, and have to merge both data before dynamic generation. This method guarantees running of other existing systems.

\subsection{Improvement of stored data quality}

As an inherent feature of this system, there is no inconsistency between ledgers and drawings even when any sort of modification has been performed. In addition, data handling will be easy because of a simple user interface. Users can verify their data modification by the generated drawing immediately. Therefore, this system provides an effective means not only to eliminate data inconsistency but also to acquire correct data.

\section{Conclusions}

We have introduced DGS which is a highly effective method to construct a computerized facility management system in railways. In addition, we introduced the above aims, fundamental concepts and merits of DGS. Moreover, we confirmed a function to register and change a facility interactively on drawings. Although most of Japanese railway companies have adopted a computerized facility management system, they still have some serious problems, such as high costs of initial data registration, inconsistency between ledgers and drawings, incomplete data integrity and insufficient maintenance ability. We are sure that DGS will contribute to eliminating these problems and improving the existing facility management systems. Some railway companies in Japan are introducing this DGS system in recent years to take advantage of the merits of DGS.

\section{References}

1) Nagata, M. and Tanaka, M.: "Facility Management System Using Facility Description Language," RTRI Report (in Japanese), Vol. 9, No. 11, pp. 43-48, 1995.

2) Satoh, N., Nagata, M., Yagi, Y., Hayashi, R. and Tanaka, M.: "PDA for Field Engineers in Railway," Proceedings of WCRR' 96, 1996.

3) Nagata, M., Hamanaka, A. and Tanaka, M.: "Development of Dynamic Generation System for Systematic Facility Drawings," RTRI Report (in Japanese), Vol. 12, No. 2, pp. 47-52, 1998.

4) Nagata, M. and Tanaka, M.: "Study on Dynamic Generation for Drawings in Facility Management System," QR of RTRI, Vol. 39, No. 4, pp. 170-175, 1998. 